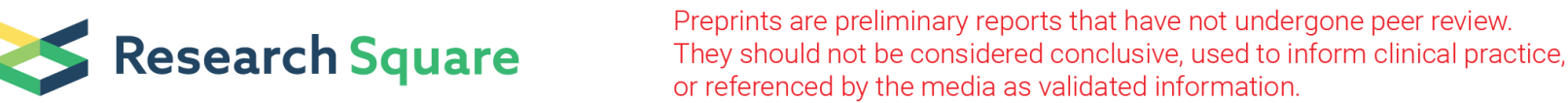

\section{Naloxone and nalmefene absorption delivered by hollow microneedles compared to intramuscular injection}

Mark G. Papich ( $\square$ Mark_Papich@ncsu.edu )

North Carolina State University https://orcid.org/0000-0002-7591-7898

Roger J. Narayan

North Carolina State University

\section{Research Article}

Keywords: microneedle, opioid, overdose, naloxone nalmefene

Posted Date: September 30th, 2021

DOI: https://doi.org/10.21203/rs.3.rs-933534/v1

License: (c) (1) This work is licensed under a Creative Commons Attribution 4.0 International License. Read Full License

Version of Record: A version of this preprint was published at Drug Delivery and Translational Research on November 24th, 2021. See the published version at https://doi.org/10.1007/s13346-021-01096-0. 


\section{Abstract}

Naloxone and nalmefene were administered to seven research Beagle dogs, (mean weight approximately $12 \mathrm{~kg}$ ) at a dose of $0.04 \mathrm{mg} / \mathrm{kg}$ and $0.014 \mathrm{mg} / \mathrm{kg}$ for naloxone and nalmefene, respectively. Each dose was administered intramuscularly (IM) with a standard IM injection and with a hollow microneedle device array using needles of $1 \mathrm{~mm}$ in length. The IM injection was delivered in the epaxial muscles, and the microneedle injection was delivered in the skin over the shoulder of each dog. Each dog received the same injections in a cross-over design. Following the injection, blood samples were collected for plasma analysis of naloxone and nalmefene by high pressure liquid chromatography with mass spectrometry detection (LCMS). The plasma sample concentrations were plotted for observed patterns of absorption and analyzed with non-compartmental pharmacokinetic methods (NCA). The results showed that the injection of naloxone from the microneedle device produced a higher peak concentration $\left(\mathrm{C}_{\text {MAX }}\right)$ by $2.15 \mathrm{x}$ compared the IM injection of the same dose, and time to peak concentration ( $\left.T_{\text {MAX }}\right)$ was similar. For the nalmefene injection, the peak was not as high (lower $\mathrm{C}_{\text {MAX }}$ ) by $0.94 \mathrm{x}$ for the microneedle injection compared to the IM injection of the same dose. The microneedle produced an exposure, measured by area under the curve (AUC)) that was $0.85 x$ and $0.58 x$ as high for naloxone and nalmefene, respectively, than the injection by the IM route. We also observed that although the dose for naloxone was approximately $3 x$ higher for naloxone compared to nalmefene, the mean peak concentration achieved from the naloxone injection was more than $12 x$ higher than the nalmefene injection. These studies were designed to test the feasibility of using the hollow microneedle array as an effective method of naloxone and nalmefene delivery for emergency treatment of opioid-induced respiratory depression (OIRD). The results of these studies will form the basis of future studies, using the dog as a model, for development of hollow microneedle microarray devices to deliver opioid antagonists for treatment of OIRD in people.

\section{Introduction}

The non-medical use of opioids, particularly among young people, is a significant public health concern in the United States and other countries. According to the U. S. Centers for Disease Control and Prevention, 841,000 people have died since 1999 from a drug overdose; in 2019, 70,630 drug overdose deaths occurred in the United States [1]. Opioids, mainly synthetic opioids such as fentanyl and fentanyl derivatives, are the main driver of drug overdose deaths, with $73 \%$ of opioid-involved overdose deaths involving synthetic opioids.

The treatment of opioid overdose consists primarily of emergency administration of the opioid competitive mu-receptor antagonist naloxone [2]. The routes of naloxone administration include intramuscular (IM) injection, subcutaneous injection, intravenous injection, or intranasal delivery (e.g., Narcan ${ }^{\circledR}$ nasal spray). IM delivery has been previously demonstrated as an effective approach for the delivery of naloxone [2]; naloxone is considered to be safer than intravenous naloxone in terms of potential exposure to blood-borne pathogens. Some training and comfort level are needed for bystander to use pre-filled hypodermic syringes for performing intravenous or IM injections. In addition, intravenous access may be difficult to obtain in individuals with prior intravenous drug use or in individuals who 
exhibit hypotension during an overdose. The cost is another limiting factor; for example, the consumer price of one $2 \mathrm{mg}$ pre-filled naloxone IM injector (Evzio ${ }^{\circledR}$ ) exceeded \$2,000 in 2019 and the consumer price one $4 \mathrm{mg}$ Narcan ${ }^{\circledR}$ nasal spray exceeded $\$ 90$ [3]; it should be noted that repeated doses may be required for treatment of a single overdose event.

Recent research efforts have described the development of alternative approaches to conventional intravenous or IM delivery. Nebulizers may be used for the treatment of opioid overdose in emergency settings; however, the disadvantage of this approach is that individuals must have spontaneous respirations for nebulizers to be effective [4]. Nebulizers are also relatively large devices that require electrical power for operating air compressors. Intranasal delivery is another alternative; however, this approach may be complicated by (a) variations in nasal anatomy due to trauma or nasal pathology as well as (b) variations in response due to mucous material, epistaxis, or upper respiratory infection [5]. Panchagnula et al. described successful transdermal delivery of naloxone to Sprague-Dawley rats via a reservoir patch; however, the slow rate of onset and the large variation in skin permeability among humans make transdermal administration via a patch unpredictable for emergent treatment [6]. Yamamoto et al. successfully utilized anodal iontophoresis patches for transdermal delivery of naloxone to murine dorsal skin and porcine ear skin [7]. They showed in vivo steady-state transdermal absorption rates of 136 and $305 \mu \mathrm{g} / \mathrm{h} / \mathrm{cm}^{2}$ for 90 and $180 \mu \mathrm{A} / \mathrm{cm}^{2}$, respectively; however, Sanaei-Zadehi indicated that this approach is inappropriate for emergent treatment because of the low delivery rate [8]. Rhodes noted another disadvantage of iontophoresis is that this approach requires a power source; concerns over reliability, cost, and lifetime of the power source may also limit the use of iontophoresis [9].

Treatment of opioid overdose and opioid-induced respiratory depresssion (OIRD) will be facilitated with devices that simplify the ease of treatment administration. Our laboratory has an on-going effort to develop a microneedle injection system that can deliver an opioid antagonist effectively, without prior training, and with minimal discomfort to the receipient. It is first necessary to demonstrate a proof-ofconcept that microneedle delivery will be absorbed systemically at a rate and extent that compares to delivery by a conventional IM injection.

Administration of naloxone is associated with restoration of spontaneous respirations in under two minutes [2]. We hypothesized that similar effects can be achieved with nalmefene (Revex). We selected nalmefene to include in these studies because it is more potent - thus allowing for smaller volumes of injection - and is potentially more effective than naloxone. Its disposition in dogs is well-known [10]; in addition, nalmefene was 4-5 times more potent than naloxone for reversing fentanyl respiratory depression [11-13]. The onset of effect of nalmefene is as rapid as naloxone; in addition, it has a significantly longer (by $2 \mathrm{x}$ ) duration of fentanyl antagonism compared to naloxone. Because of high potency and solubility, nalmefene can be delivered in a small volume. Although nalmefene injection was withdrawn by the sponsor in 1995, it was for commercial (not safety) concerns [13].

Our hypothesis is that an injection of either naloxone or nalmefene administered with a hollow microneedle array will produce plasma drug concentrations as high, or higher, than conventional IM 
injections. Our long-term goal is to use these data to engineer a microdevice that can be easily administered to people experiencing an opioid overdose when trained bystanders are unavailable. Microneedle device technology is well-known and has previously been demonstrated via in vitro and in vivo studies [14-20]. Since naloxone and nalmefene have short half-lives, treated individuals can be brought to medical care after 1-3 treatments from hollow microneedle arrays. Moreover, hollow microneedle arrays can be inexpensively produced from metal and/or polymer components.

We selected dogs for these studies because of prior experience with canine models in our laboratory and ease of handling and administration. Although other studies have used pig skin as a model for comparison to human skin [21,22], we have substantial evidence that dog skin in the shoulder area (our site of administration for these studies) will be of comparable thickness to the skin on the inner forearm of people. Dogs serve as a suitable model in these studies with hollow microneedle array injection because the thickness of stratum corneum inner human forearm skin is $15 \mu \mathrm{m}$ and thickness of dog skin over the shoulder approximately $12 \mu \mathrm{m}$ [23-25].

\section{Materials And Methods}

\section{Animals:}

Seven healthy research dogs (Beagles) weighing approximately $12 \mathrm{~kg}$, were obtained and maintained in the Laboratory Animal Resources Facility at the NC State University College of Veterinary Medicine. We used a cross-over design in which each animal served as its own control. The dogs were accurately weighed and examined to ensure that they are healthy before initiation of the study. After a brief period of acclimatization, the dogs were sedated using an IM injection of dexmedetomidine ( $8 \mu \mathrm{g} / \mathrm{kg})$. While sedated, intravenous catheters were inserted in the jugular vein and secured in place to facilitate easy access to blood sample collection. The catheters were periodically rinsed with saline to maintain patency throughout the study. The dogs were allowed to fully recover from the sedation and catheter placement procedure for at least sixteen hours prior to the scheduled study. The study was approved by the college's Institutional Animal Care and Use Committee (IACUC) prior to the start of the project (IACUC approval \# 19-548).

\section{Study Design:}

The absorption of naloxone and nalmefene from the hollow microneedle array compared to an IM injection was determined using a two-period, two-treatment, cross-over design for each drug. This design compared the pharmacokinetics of conventional IM naloxone delivery (naloxone hydrochloride injection, concentration, $0.4 \mathrm{mg} / \mathrm{mL}$ ) at a dose of $0.04 \mathrm{mg} / \mathrm{kg}$ to the pharmacokinetics of naloxone delivery from the hollow microneedle array using the same dose and formulation. The volume administered was 0.1 $\mathrm{mL} / \mathrm{kg}$. The same administration was repeated with nalmefene using a dose of $0.014 \mathrm{mg} / \mathrm{kg}$ and a concentration of $0.15 \mathrm{mg} / \mathrm{mL}$ nalmefene, which was prepared in our laboratory using commercially available powder (TOCRIS, www.tocris.com). The doses selected for injection were derived from studies showing that this dose is adequate to reverse opioid overdose in dogs [11-12, 26-28]. 
The hollow microneedle array was applied to the skin over the shoulder (after clipping), pressed into the skin, and administered with a $3 \mathrm{~mL}$ syringe (approximately $1.2 \mathrm{~mL}$ per dog). The skin over the shoulder was selected because it is most similar in thickness to human forearm skin. The IM injection was administered in the epaxial muscle group over the back of each dog at the same dose.

Each treatment was applied to all of the dogs in a crossover manner; a washout period was used to separate each study. The washout period consisted of at least ten half-lives to ensure elimination of each dose. After each treatment, blood samples were collected from the jugular catheter to characterize the pharmacokinetics. Approximately $3 \mathrm{~mL}$ blood samples were collected, prior to administration, and 5, 10, 15 , and 30 minutes as well as at 1,2,3,4, and 6 hours. Blood samples were centrifuged to harvest plasma and stored in a $-80^{\circ} \mathrm{C}$ freezer prior to analysis.

\section{Microneedle Device:}

The hollow microneedle array is a $3 \times 3$ microneedle array with a luer-slip female hub connectible to typical syringe. (Micropoint Technologies Pte Ltd., Pioneer Junction, Singapore) (Fig. 1). The height of the needle is designed to penetrate the stratum corneum and epidermis, but not deep enough to penetrate the thickness of the dermis [25]. The surface features of the hollow microneedle array were evaluated using a VKx1100 confocal laser scanning microscope (Keyence, Osaka, Japan). The microstructural features of the microneedles in the hollow microneedle array examined using scanning electron microscopy (SEM) with a Model S-3200N instrument (Hitachi, Tokyo, Japan) (Fig. 2).

\section{Sample Analysis:}

The plasma samples were analyzed for naloxone and nalmefene using high-performance liquid chromatography (HPLC) with mass spectrometry detection (LC-MS) in the Clinical Pharmacology Laboratory at North Carolina State University using other studies as a guide [28, 29].

The HPLC system (1260 series system; Agilent Technologies) consisted of a quaternary solvent delivery system with a flow rate of $0.8 \mathrm{~mL} / \mathrm{min}$ and autosampler. Detection was accomplished with a mass spectrometer (LC/MSD iQ Mass Selective Detector, Agilent Technologies, Santa Clara, CA) in the positive mode with a mass/charge ratio $(\mathrm{m} / \mathrm{z})$ of 328 and 340 for naloxone and nalmefene, respectively. Chromatograms were integrated with the Agilent software (OpenLAB software, Agilent Technologies, Santa Clara, CA). A C18 column (Zorbax XDB Eclipse C18, 4.6 X 15-cm column, Agilent Technologies, Santa Clara, $\mathrm{CA})$ was used and maintained at a constant temperature $\left(35^{\circ} \mathrm{C}\right)$. The mobile phase consisted of $50 \% 0.025 \mathrm{M}$ ammonium acetate in water and $50 \%$ acetonitrile. The analytical reference standard for naloxone was obtained from the United States Pharmacopeia (Rockville, MD), and the analytical reference standard for nalmefene was obtained from AdooQ (AdooQ Bioscience LLC, Irvine, $\mathrm{CA})$ These reference standards were used to make a stock solution, which was used to fortify blank plasma harvested from blood samples collected from the dogs prior to treatment (control plasma). The stock solution was sealed and stored in the dark in a refrigerator. The calibration curve for each drug consisted of 7 standard solutions with concentrations that ranged from 1 to $1,000 \mathrm{ng} / \mathrm{mL}$ and included a 
blank $(0 \mu \mathrm{g} / \mathrm{mL})$ sample. The blank sample was used to detect interfering peaks that eluted into the window of the chromatographic peak of interest and to measure background interference. The calibration curve was accepted if the linear coefficient of determination $\left(R^{2}\right)$ was $\geq 0.99$ and the calibration curve concentrations could be back calculated to within $\leq 15 \%$ of the true concentrations of the standard solutions.

All plasma, calibration, quality control, and blank plasma samples were prepared in an identical manner. Four hundred microliters of each plasma sample were added to a solid-phase extraction cartridge (Oasis ${ }^{\circledR}$ PRiME HLB Extraction Cartridge, $30 \mathrm{mg}$ ) (Waters Corporation, Milford, MA). The sample from the cartridge was collected in a clean glass tube by elution with $1 \mathrm{~mL}$ of $100 \%$ methanol. The eluted samples were evaporated to yield a dry residue by heating the tubes at $40^{\circ} \mathrm{C}$ under airflow. The residue in each tube was reconstituted by addition of $200 \mu \mathrm{L}$ of the mobile phase; the solution was briefly vortexed and then transferred to an HPLC injection vial. A $20-\mu \mathrm{L}$ aliquot of each sample was used for injection into the HPLC system. Fresh calibration and blank samples were prepared for analysis each day. The limit of quantification (LOQ) for naloxone and nalmefene were based on the basis of the signal:noise ratio in accordance with published guidelines [30].

\section{Pharmacokinetic Analysis:}

The pharmacokinetic analysis used a simple non-compartmental analysis, with the area-under-the-curve (AUC) estimated from the summation of trapezoids and the terminal rate estimated from regression of the terminal plasma concentration points after the peak ( $\left.\mathrm{C}_{\text {MAX }}\right)$ concentration. The $\mathrm{C}_{\text {MAX }}$ and time to peak concentration ( $\left.T_{\text {MAX }}\right)$ were estimated from the data. The terminal half-life was calculated from $L n$ $0.5 /$ terminal rate, in which Ln 0.5 is the natural logarithm of one-half. All calculations were performed with Phoenix $\AA$ pharmacokinetic modeling software (WinNonlin ${ }^{\circledR}$, Certara Company, St. Louis, MO, Version 8.4). Relative bioavailability of microneedle vs IM injection was calculated from the AUC and $\mathrm{C}_{\mathrm{MAX}}$ ratios. Systemic clearance and volume of distribution were not reported because there was no accompanying intravenous dose.

\section{Results And Discussion}

Figure 1 (a) shows a $3 \mathrm{~d}$ representation of a hollow microneedle array. The component needles are $1 \mathrm{~mm}$ in length and are arranged in a $3 \times 3$ array; the needle pitch, the distance between the centers of the needles, is $3.5 \mathrm{~mm}$. Figure 1 (b) shows a side view optical micrograph of a single microneedle within the hollow microneedle array, and Fig. 1 (c) shows a top view optical micrograph of a single microneedle within the hollow microneedle array. The needle outer diameter is $250 \mu \mathrm{m}$, and the needle inner diameter is $150 \mu \mathrm{m}$. Figure 2 (a) shows a scanning electron micrograph of single microneedle within the hollow microneedle array, Fig. 2 (b) shows a scanning electron micrograph of the tip of a single microneedle within the hollow microneedle array, and Fig. 2 (c) shows a top view scanning electron micrograph of a single microneedle within the hollow microneedle array. The needle exhibits a circular bore and a sharp tip. 
The plasma drug concentrations for naloxone and nalmefene from each route of administration to dogs are shown in Fig. 3 (top and bottom panel). As observed in the figure, the microneedle injection produced high plasma naloxone concentrations rapidly that were higher than the IM injection of the same dose, but the absorption was more delayed and lower from the nalmefene microneedle injection. The duration of detectable concentrations from the nalmefene injection was much shorter, compared to the naloxone injection because the assay limit was reached quickly at these low nalmefene concentrations. As seen by the error bars on the figures, there was considerable variability in the concentrations for both drugs.

The pharmacokinetic results are shown in Tables 1 and 2. For the naloxone injection, the peak was higher from the microneedle injection, as seen by the $\mathrm{C}_{\text {MAX }}$ values, with the microneedle injection producing a peak that was a mean of $2.15 \mathrm{x}$ higher than the IM injection. The extent of absorption, shown by the area under the curve (AUC) values was not as high from the microneedle injection, which was likely due to the shorter half-life (mean of $0.46 \mathrm{hr}$ and $1.2 \mathrm{hr}$ from the microneedle and IM injection, respectively).

Table 2

Pharmacokinetic parameters after injection of nalmefene to dogs at a dose of $0.014 \mathrm{mg}$, either intramuscularly or by hollow microneedle array.

\begin{tabular}{|c|c|c|c|c|c|}
\hline \multirow[b]{2}{*}{ Parameter } & \multirow[b]{2}{*}{ Units } & \multicolumn{2}{|c|}{ Intramuscular } & \multicolumn{2}{|c|}{ Microneedle } \\
\hline & & Mean & Std. Dev. & Mean & Std. Dev. \\
\hline AUC (0 to $\mathrm{Cn})$ & hr*ng/mL & 6.72 & 2.73 & 4.72 & 2.64 \\
\hline AUC (0 to infinity) & $h r^{*} n g / m L$ & 8.02 & 3.61 & 5.08 & 2.71 \\
\hline $\mathrm{C}_{\text {MAX }}$ & $\mathrm{ng} / \mathrm{mL}$ & 4.71 & 1.18 & 3.80 & 2.64 \\
\hline Half-life $\left(t^{1} 1 / 2\right)$ & $\mathrm{hr}$ & 1.39 & 1.18 & 0.85 & 0.59 \\
\hline Terminal rate & $1 / \mathrm{hr}$ & 1.01 & 0.80 & 1.09 & 0.56 \\
\hline MRT & $\mathrm{hr}$ & 2.00 & 1.51 & 1.59 & 0.97 \\
\hline $\mathrm{T}_{\text {MAX }}$ & $\mathrm{hr}$ & 0.33 & 0.17 & 0.45 & 0.43 \\
\hline Fraction $\mathrm{C}_{\mathrm{MAX}}$ & & & & 0.94 & 0.75 \\
\hline Fraction AUC & & & & 0.58 & 0.12 \\
\hline
\end{tabular}

Legend: $A U C$ ( 0 to $\mathrm{Cn}$ ), area under the curve from time 0 to the last measured time point (Cn); AUC (0 to infinity), the area under the curve from time 0 to infinity; $\mathrm{C}_{\mathrm{MAX}}$, maximum (peak) plasma concentration; half-life ( $\left(1 \frac{1}{2}\right)$ terminal half-life; MRT, mean residence time; $\mathrm{T}_{M A X}$, time to peak $\left(\mathrm{C}_{\text {MAX }}\right)$ concentration; Fraction $\mathrm{C}_{\text {MAX }}$, the ratio of microneedle $\mathrm{C}_{\text {MAX }}$ concentration to intramuscular $\mathrm{C}_{\text {MAX }}$ concentration; Fraction AUC, the the ratio of microneedle AUC to intramuscular AUC concentration. 
For the nalmefene injection the peak ( $\left.\mathrm{C}_{\mathrm{MAX}}\right)$ was slightly lower from the microneedle injection with a mean difference of $0.94 x$ lower, compared to the IM injection. The extent of absorption was considerably lower from the microneedle injection, with a mean relative fraction absorbed of 0.58 . Compared to naloxone, the nalmefene concentrations were proportionally much lower for nalmefene. Although the dose of naloxone was approximately $3 x$ higher than the nalmefene dose, the difference in $\mathrm{C}_{\text {MAX }}$ was over $12 x$ higher for naloxone after the microneedle injection and approximately $5 x$ higher after the IM injection. The reasons for these differences are undetermined. According to the NIH U.S. National Library of Medicine (https://chem.nlm.nih.gov/chemidplus/) naloxone and nalmefene have similar lipid solubility values (LogP) but naloxone is much more soluble than nalmefene by a factor of approximately 10x. For a drug to be readily absorbed into plasma after an injection, solubility is an important factor and it is possible that this accounts for the observed differences in relative plasma concentrations from the nalmefene injections compared to naloxone injections. In a study in people [33] after a nalmefene hydrochloride injection of $0.019 \mathrm{mg} / \mathrm{kg}$ (mean) IM, the $\mathrm{C}_{\text {MAX }}$ was $1.53 \mathrm{ng} / \mathrm{mL}$ at a $T_{\text {MAX }}$ of 0.33 hours. Thus, the IM absorption of nalmefene we observed in dogs was actually higher than reported for people.

This is the first study to examine the disposition of nalmefene and naloxone in dogs delivered with a hollow microneedle array. The objective of this study was to characterize absorption, using the dog as our model, for future development of a hollow microneedle array that will deliver naloxone or nalmefene to humans for emergency treatment of opioid-induced respiratory depression (OIRD).

We used the dog as our model because they will be the basis of our future assessment. The dog is an ideal model animal in these studies because (a) they can be easily instrumented and sampled for pharmacokinetic studies, (b) their heart rate, respiratory rate, blood oxygenation, and other parameters are easily monitored during assessment of the hollow microneedle arrays, (c) they have a predictable response to an overdose of fentanyl [11], and (d) their skin stratum corneum thickness is similar to the forearm of humans, thus providing an accurate assessment of intradermal delivery with these microneedles that are $1 \mathrm{~mm}$ in length. Our laboratory at the College of Veterinary Medicine has extensive experience handling and instrumenting dogs for pharmacology studies.

We selected naloxone for our studies because it is the standard method of opioid overdose emergency treatment [2]. We showed that the microneedle injections of naloxone produce rapid plasma drug concentrations in dogs compared to a traditional IM injection at the same dose with comparable rate of absorption (Fig. 3, Table 1). The dose we used $(0.04 \mathrm{mg} / \mathrm{kg})$ is the standard dose in dogs for opioid reversal [12]. We selected nalmefene because it had potential for future development owing to its $4-5 x$ greater potency and $2 x$ longer duration than naloxone [11-13]. The dose used in our study $(0.014 \mathrm{mg} / \mathrm{kg})$ was lower than the naloxone dose to account for its higher potency. However, we did not anticipate the lower relative absorption from the nalmefene injection, which may limit the potential for this application in the future.

The mean peak concentrations $\left(\mathrm{C}_{\mathrm{MAX}}\right)$ were $2.15 \mathrm{x}$ higher from the microneedle injection of naloxone compared to the IM injection, but the mean relative bioavailability (measured by AUC ratios) was less (by 
$0.85 x$ ) for the microneedle injection (Table 1 ). This was likely due to a faster half-life associated with the microneedle injection. For rapid treatment of opioid overdose, a rapid high peak concentration is more critical to a successful outcome than the AUC.

We observed substantial variation in on our data as seen by the error bars in Fig. 3 as well as the variation in Tables 1 and 2. This result may be associated with our observation that some of the dogs pulled away from the microneedle injection because of a slight "sting" at the time of injection; this reaction may have produced an incomplete or variable injection volume. We attribute this response to the formulation of each drug. Both drugs are formulated as hydrochloride salts $(\mathrm{HCl})$. It has been our observation from clinical use of medications in dogs that drugs formulated in hydrochloride can cause an initial sting or discomfort upon injection because of the low $\mathrm{pH}$. We do not anticipate such a problem when used to treat an opioid overdose because the individual will be under the strong influence of a potent opioid at the time of treatment.

Pharmacokinetic data for naloxone or nalmefene in dogs is somewhat limited. Naloxone is characterized by a short half-life and a high hepatic clearance that exceeds hepatic blood flow [28]. Pace et al. studied naloxone at a high dose of $5 \mathrm{mg} / \mathrm{kg}$ IV in dogs; however, they used a radioimmunoassay, which has considerable cross-reactivity with other opioids and metabolites. In that study, they described a terminal half-life of approximately 71 minutes [31].

Other studies used a more specific HPLC assay with electrochemical detection or HPLC coupled with mass spectrometry (LCMS), as performed in this study. Garrett et al. used an electrochemical detection approach and showed that there was no pharmacokinetic dose-dependency when they compared 10-fold differences in doses in dogs of 0.47 and $4.7 \mathrm{mg} / \mathrm{kg} \mathrm{IV} \mathrm{[32].} \mathrm{The} \mathrm{terminal} \mathrm{half-life} \mathrm{(mean)} \mathrm{was} 55.6$ minutes with a high clearance. Wahler et al. showed a terminal half-life (mean) of 37 minutes from an IV injection of $0.04 \mathrm{mg} / \mathrm{kg}$, the same dose used in our study [28]. When they gave the same dose intranasal, the bioavailability was only $32 \%$. Our study also showed that the disposition of these drugs in dogs was characterized by a rapid terminal half-life in most dogs (Fig. 1, Tables 2 and 3) with a mean of $1.2 \mathrm{hr}$ for naloxone given IM and $0.46 \mathrm{hr}$ after the microneedle injection. Therefore, these values are in a similar range as other studies. Nalmefene also had a rapid half-life of approximately $1 \mathrm{hr}$ for both routes of administration. The measureable concentrations were also short-lived because the values quickly fell below our assay limits.

Other than the slight discomfort from the microneedle injection, as noted above, there were no adverse effects observed in the dogs after these injections. We observed that the microneedle array produced a slight imprint on the clipped skin of these dogs; this feature was only temporary and resolved a few hours after injection.

\section{Conclusions}

We showed that delivery of naloxone, when administered at a dose anticipated for the reversal of OIRD, was absorbed rapidly and with a higher peak concentration than a comparable injection delivered by the 
conventional IM route in dogs. Thus, for naloxone, the microneedle delivery may be a more effective initial treatment for OIRD compared to IM administration. However, the microneedle injection of

nalmefene was not absorbed as well, compared to the IM injection with a lower $\mathrm{C}_{\mathrm{MAX}}$ and lower relative bioavailability. In addition, the proportional difference in absorption, after accounting for differences in $\mathrm{mg} / \mathrm{kg}$ doses was much lower for nalmefene compared to naloxone. These studies establish the proof in principle of opioid overdose delivery treatment using a hollow microneedle array, and the use of dogs as a suitable model for future development of this approach.

\section{Declarations}

\section{ACKNOWLEDGEMENTS}

The authors thank Delta R. Dise of the North Carolina State University Clinical Pharmacology Laboratory for her assistance with performing the drug assays. We thank the staff of the North Carolina State University Central Procedures Laboratory for their technical assistance. The investigators would like to acknowledge the National Institute on Drug Abuse (Grant \#5-R21A-DA040916-02) for their support of this research.

\section{CONFLICTS OF INTEREST STATEMENT}

The authors declare no conflicts of interest or competing interests.

\section{ETHICAL STATEMENT}

- Ethics approval and consent to participate: This study was performed in dogs. We obtained an approved IACUC protocol prior to the study.

- Consent for publication: All authors provided consent for publication.

- Availability of data and materials: All the data and materials used are freely available.

- Competing interests: There are no competing interests for this study and no prior publication.

- Funding: The investigators would like to acknowledge the National Institute on Drug Abuse (Grant \#5-R21A-DA040916-02) for their support of this research.

- Authors' contributions: Both authors contributed to the manuscript preparation and editing. Dr. Papich performed the animal studies and pharmacokinetic analysis; Dr. Narayan provided technical input, review of manuscript and assisted in the design of the study.

- Acknowledgements: The authors thank Delta R. Dise of the North Carolina State University Clinical Pharmacology Laboratory for her assistance with performing the drug assays. We thank the staff of the North Carolina State University Central Procedures Laboratory for their technical assistance.

- Authors' information (optional): The authors are both on the faculty of North Carolina State University 


\section{References}

1. Centers for Disease Control and Prevention. Wide-ranging online data for epidemiologic research (WONDER). Atlanta, GA: CDC, National Center for Health Statistics; 2020. Available at http://wonder.cdc.gov.

2. Boyer EW. Management of opioid analgesic overdose. N Engl J Med. 2012;367(2):146-55.

3. Naloxone Pricing in the Community. (As of January 2019). Available at https://www.umassmed.edu/globalassets/office-of-undergraduate-medical-educationmedia/naloxone-pricing-105.pdf.

4. Mycyk MB, Szyszko AL, Aks SE. Nebulized naloxone gently and effectively reverses methadone intoxication. J Emerg Med. 2003;24:185-7.

5. Ashton H. Intranasal naloxone in suspected opioid overdose. Emerg Med J. 2006;23:221-3.

6. Panchagnula R, Bokalial R, Sharma P, Khandavilli S. Transdermal delivery of naloxone: Skin permeation, pharmacokinetic, irritancy and stability studies. Int J Pharm. 2005;293:213-23.

7. Yamamoto R, Takasuga S, Yoshida Y, Mafune S, Kominami K, Sutoh C, Kato Y, Yamauchi M, Ito M, Kanamura K, Kinoshita M. In vitro and in vivo transdermal iontophoretic delivery of naloxone, an opioid antagonist. Int J Pharm. 2012;422:132-8.

8. Sanaei-Zadeh $\mathrm{H}$. Is transdermal iontophoretic delivery of naloxone sufficient for the management of intoxication in opioid-overdosed patients? Int J Pharm. 2012;428:187.

9. Rhodes WE III. Iontophoretic drug delivery: New advantages revitalize an established technology. Drug Delivery Technology. 2002;2:34-7.

10. Murthy SS, Mathur C, Kvalo LT, Lessor RA, Wilhelm JA. Disposition of the opioid antagonist, nalmefene, in rat and dog. Xenobiotica. 1996;26(7):779-92.

11. Veng-Pedersen P, Wilhelm JA, Zakszewski TB, Osifchin E, Waters SJ. Duration of opioid antagonism by nalmefene and naloxone in the dog: an integrated pharmacokinetic/pharmacodynamic comparison. Journal of pharmaceutical sciences. 1995;84(9):1101-6.

12. Wilhelm JA, Veng-Pedersen P, Zakszewski TB, Osifchin E, Waters SJ. Duration of opioid antagonism by nalmefene and naloxone in the dog. A nonparametric pharmacodynamic comparison based on generalized cross-validated spline estimation. Int J Clin Pharmacol Ther. 1995;33(10):540-5.

13. France CP, Ahern GP, Averick S, Disney A, Enright HA, Esmaeli-Azad B, Federico A, Gerak LR, Husbands SM, Kolber B, Lau EY. Countermeasures for preventing and treating opioid overdose. Clin Pharmacol Ther. 2021;109(3):578-90.

14. Boehm RD, Miller PR, Daniels J, Stafslien S, Narayan RJ. Inkjet printing for pharmaceutical applications. Mater Today. 2014;17:247-52.

15. Boehm RD, Miller PR, Schell WA, Perfect JR, Narayan RJ. Inkjet printing of amphotericin b onto biodegradable microneedles using piezoelectric inkjet printing. JOM. 2013;65:525-33.

16. Boehm RD, Miller PR, Singh R, Shah A, Stafslien S, Daniels J, Narayan RJ. Indirect rapid prototyping of antibacterial acid anhydride copolymer microneedles. Biofabrication. 2012;4:011002. 
17. Gittard SD, Nguyen A, Obata K, Koroleva A, Narayan RJ, Chichkov BN. Fabrication of microscale medical devices by two-photon polymerization with multiple foci via a spatial light modulator. Biomedical Optics Express. 2011;2:3167-78.

18. Koroleva A, Schlie S, Fadeeva E, Gittard SD, Ovsianikov A, Koch J, Narayan RJ, Chichkov B. Microreplication of laser-fabricated surface and three-dimensional structures. J Opt. 2010;12:124009.

19. Narayan RJ, Doraiswamy A, Chrisey DB, Chichkov BN. Medical prototyping using two photon polymerization. Mater Today. 2010;13:44-50.

20. Zhang J, Wang Y, Jin JY, Degan S, Hall RP, Boehm RD, Jaipan P, Narayan RJ. Use of drawing lithography-fabricated polyglycolic acid microneedles for transdermal delivery of itraconazole to a human basal cell carcinoma model regenerated on mice. JOM. 2016;68:1128-33.

21. Riviere JE, Monteiro-Riviere NA. The isolated perfused porcine skin flap as an in vitro model for percutaneous absorption and cutaneous toxicology. CRC Critical Reviews in Toxicology. 1991;21:329-44.

22. Schmook FP, Meingassner JG, Billich A. Comparison of human skin or epidermis models with human and animal skin in in-vitro percutaneous absorption. Int J Pharm. 2001;215:51-6.

23. Rushmer RF, Buettner KJ, Short JM, Odland GF. The skin. Science. 1966;154:343-8.

24. Holbrook KA, Odland GF. Regional differences in the thickness (cell layers) of the human stratum corneum: an ultrastructural analysis. Journal of Investigative Dermatology. 1974;62(4):415-22.

25. Monteiro-Riviere NA, Bristol DG, Manning TO, Rogers RA, Riviere JE. Interspecies and interregional analysis of the comparative histologic thickness and laser Doppler blood flow measurements at five cutaneous sites in nine species. Journal of Investigative Dermatology. 1990;95(5):582-6.

26. Copland VS, Haskins SC, Patz J. Naloxone reversal of oxymorphone effects in dogs. Am J Vet Res. 1989;50(11):1854-8.

27. Freise KJ, Newbound GC, Tudan C, Clark TP. Naloxone reversal of an overdose of a novel, long-acting transdermal fentanyl solution in laboratory Beagles. J Vet Pharmacol Ther. 2012;35:45-51.

28. Wahler BM, Lerche P, Ricco Pereira CH, Bednarski RM, KuKanich B, Lakritz J, Aarnes TK. Pharmacokinetics and pharmacodynamics of intranasal and intravenous naloxone hydrochloride administration in healthy dogs. Am J Vet Res. 2019 Jul;80(7):696-701.

29. Albeck H, Woodfield S, Kreek MJ. Quantitative and pharmacokinetic analysis of naloxone in plasma using high-performance liquid chromatography with electrochemical detection and solid-phase extraction. J Chromatogr B Biomed Sci Appl. 1989;488(2):435-45.

30. United States Pharmacopeia. Validation of Compendial Procedures. USP General Chapter, $<1225>$ United States Pharmacopeia, USP-43, NF-38, Rockville, MD. 2021.

31. Pace NL, Parrish RG, Lieberman MM, Wong KC, Blatnick RA. Pharmacokinetics of naloxone and naltrexone in the dog. J Pharmacol Exp Ther. 1979;208(2):254-6. 
32. Garrett ER, Chyishyu W, Ulubelen A. Pharmacokinetics of morphine and its surrogates VIII: naloxone and naloxone conjugate pharmacokinetics in dogs as a function of dose and as affected by simultaneously administered morphine. J Pharm Sci. 1986;75(12):1127-36.

33. Krieter P, Gyaw S, Crystal R, Skolnick P. Fighting fire with fire: development of intranasal nalmefene to treat synthetic opioid overdose. J Pharmacol Exp Ther. 2019;371(2):409-15.

\section{Tables}

Due to technical limitations, Table 1 is only available as a download in the Supplemental Files section.

\section{Figures}
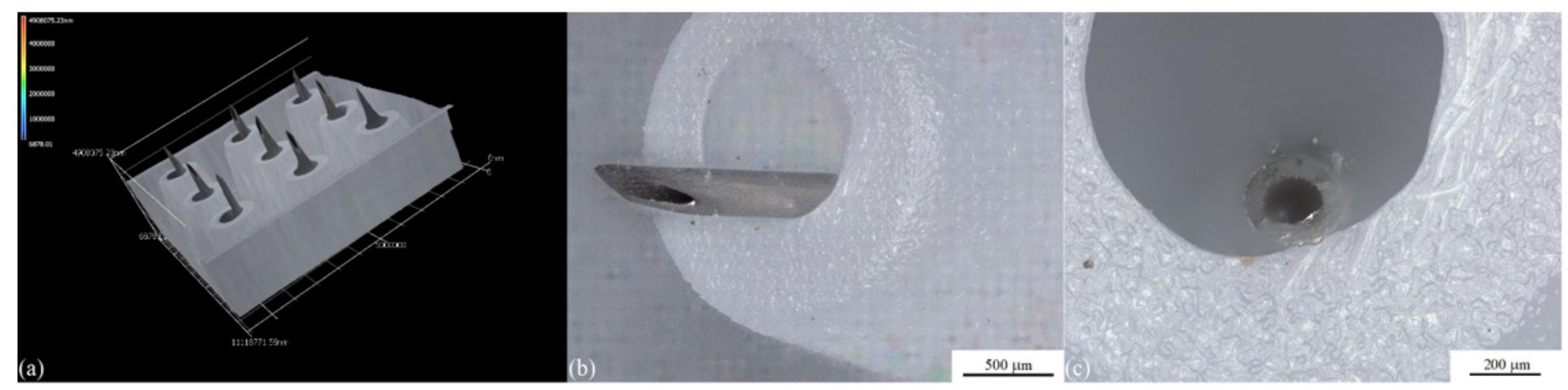

\section{Figure 1}

(a) 3d representation of a hollow microneedle array. (b) Side view optical micrograph of a single microneedle within the hollow microneedle array. (b) Top view optical micrograph of a single microneedle within the hollow microneedle array.

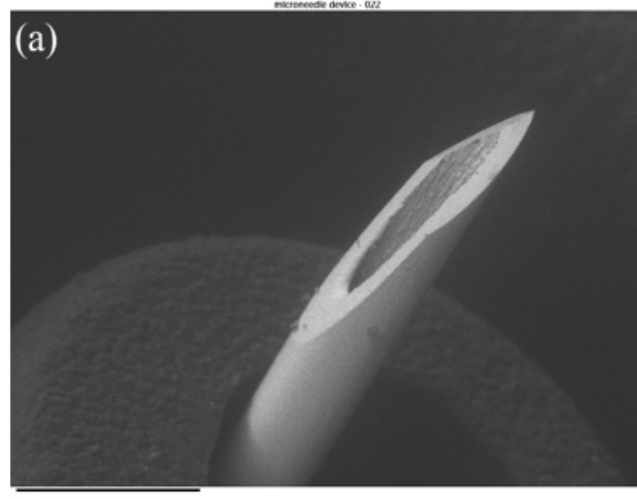

$500 \mu \mathrm{m}$

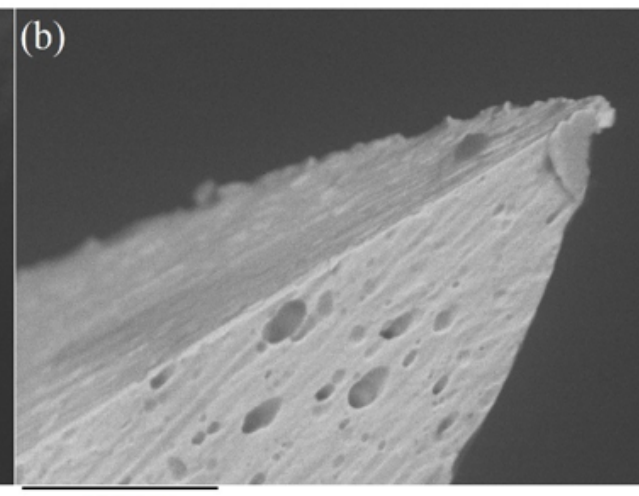

$25 \mu \mathrm{m}$

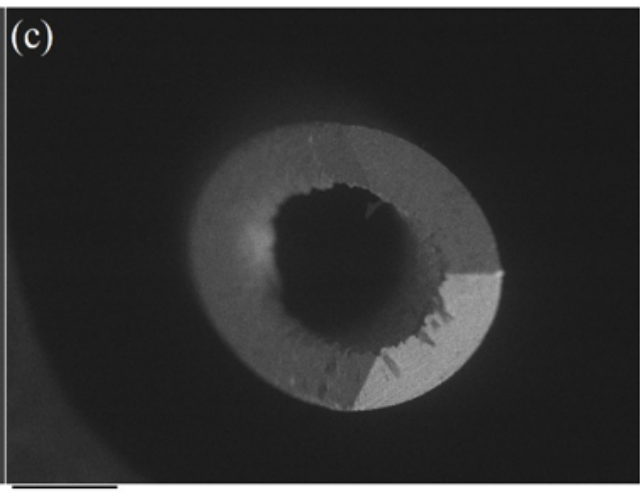

$100 \mu \mathrm{m}$

\section{Figure 2}

(a) Scanning electron micrograph of a single microneedle within the hollow microneedle array. (b) Scanning electron micrograph of the tip of a single microneedle within the hollow microneedle array. (c) Top view scanning electron micrograph of a single microneedle within the hollow microneedle array. 


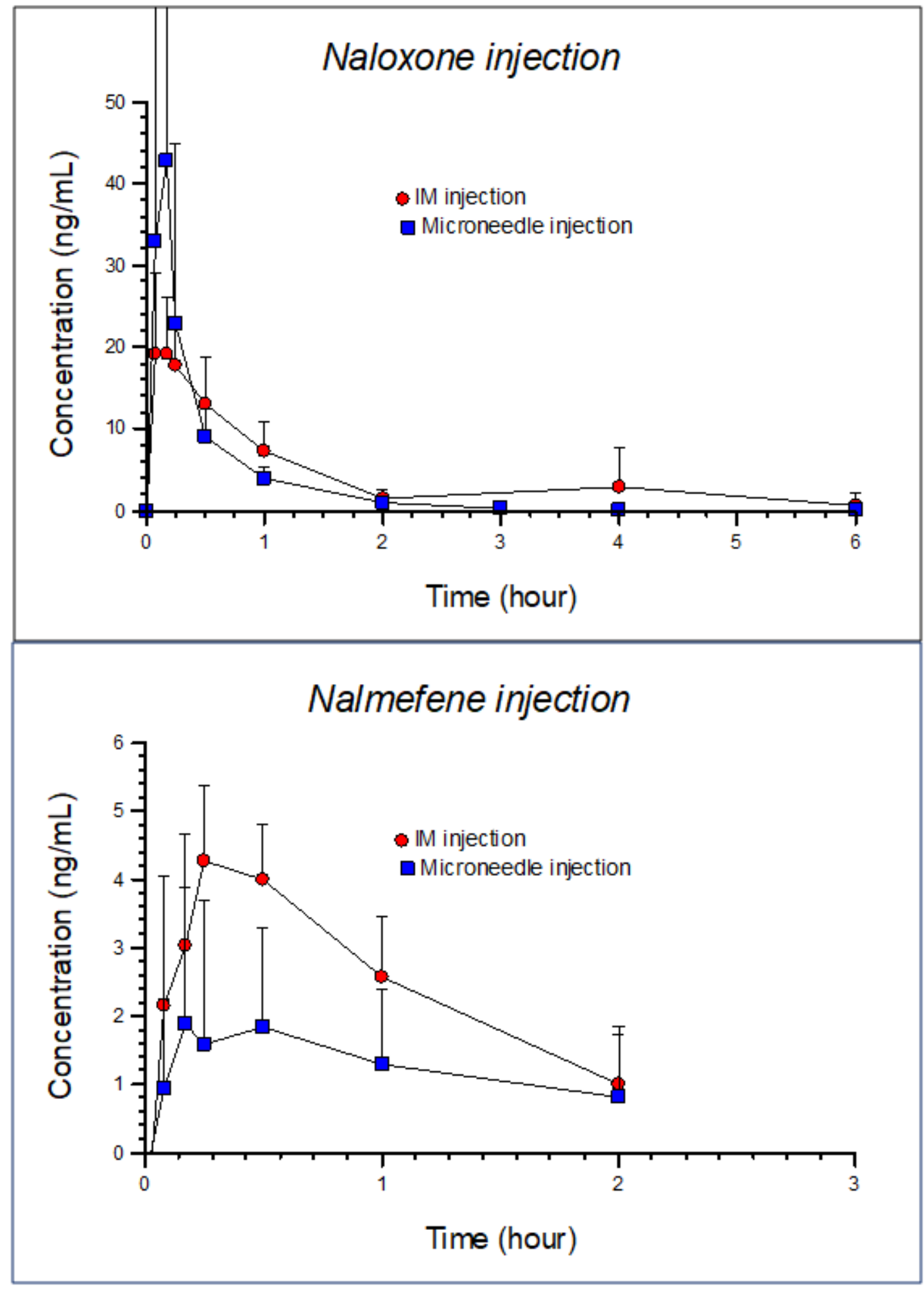

Figure 3

Plasma concentrations of naloxone (top panel) and nalmefene (bottom panel) in seven dogs administered a dose of $0.04 \mathrm{mg} / \mathrm{kg}$ (naloxone) or $0.014 \mathrm{mg} / \mathrm{kg}$ (nalmefene) by intramuscular injection (solid round symbol) or hollow microneedle array injection (solid square symbol). Each point represents the mean (+/- standard deviation). 


\section{Supplementary Files}

This is a list of supplementary files associated with this preprint. Click to download.

- TABLE1.docx

- GraphicalAbstract.tif 